\title{
Progress on an Ion Cyclotron Range of Frequency System for DEMO
}

\author{
J.-M. Noterdaeme' ${ }^{13}$, A. Messiaen' ${ }^{2}$ R. Ragona ${ }^{23}$, W. Zhang', \\ A. Bader ${ }^{1}$, F. Durodié 2 , U. Fischer ${ }^{4}$, T. Franke ${ }^{15}$, E. Smigelskis ${ }^{1}, J^{\prime}$ Ongena ${ }^{2}$, M.Q. Tran ${ }^{6}$, \\ D. Van Eester ${ }^{2}$, M. Van Schoor ${ }^{2}$ \\ ${ }^{1}$ Max-Planck-Institut für Plasmaphysik, Boltzmannstr. 2, D-85748 Garching, Germany \\ ${ }^{2}$ LPP-ERM/KMS, B-1000 Brussels, Belgium \\ ${ }^{3}$ Applied Physics Department, Ghent University, B-9000 Gent, Belgium \\ ${ }^{4}$ Karlsruhe Institute of Technology (KIT), Kaiserstr. 12, 76131Karlsruhe, Germany \\ ${ }^{5}$ EUROfusion Consortium, Boltzmannstr. 2, D-85748 Garching, Germany \\ ${ }^{6}$ Swiss Plasma Center, Ecole Polytechnique Federale de Lausanne, 1015 Lausanne, Switzerland
}

\begin{abstract}
An Ion Cyclotron Range of Frequency (ICRF) system can provide power for a number of tasks, experimentally verified on present machines: heating and current drive, first wall conditioning, plasma startup, removing central impurities, controlling sawteeth and current ramp down assist. The system has a high plug-to-power efficiency and most of the components external to the machine are sturdy, with industrial steady state capability.

Traditional ICRF antenna systems are often characterized by a high operating voltage and high power density. Low power density and low voltage however provides a bonus in terms of reliability. Therefore, travelling wave type antennas have been proposed [1]. They can be integrated in the blanket and use only a limited number of feeders. The effect on the tritium breeding ratio of such an antenna incorporated in the blanket, including the feeders, is small. The $\mathrm{k}_{/ /}$spectrum is peaked and the dominant $\mathrm{k}_{/ /}$value can be made optimized for coupling and bulk absorption, while avoiding the generation of coaxial modes in the edge. The coupling can be further enhanced with gas puffing near the antenna. Assuming the ITER-2010-low density profile, 50 MW can be coupled with a voltage on the antenna components of about $15 \mathrm{kV}$.
\end{abstract}

Keywords: ICRF, DEMO, travelling wave antenna, tritium breeding ratio

\section{Introduction}

The power density of a number present ICRF antenna is given in Table 1. Note that some of them were achieved in L-mode (TS, ILA), or in limiter configuration (JET $\mathrm{A} 0)$. Using the power density assumed for ITER in the worst case $\left(3.3 \mathrm{MW} / \mathrm{m}^{2}\right)$, about $15 \mathrm{~m}^{2}$ would be needed to couple $50 \mathrm{MW}$ in DEMO. If the antenna were located in a port to house both the antenna and all the feeders (which is equivalent to removing the total depth of the breeding blanket), then this would lead to a typical reduction in the Tritium Breeding Ratio of $3.8 \%$. The alternative is to use antenna that remove only a small depth of the breeding blanket (thus keeping a large fraction of the Tritium breeding in this area) and to reduce the number of feeders to a minimum (where the breeding is eliminated over the whole depth). Travelling wave array (TWA) type antennas are such antenna types that are not localized in ports. They combine low power density and load resilience with a reduced number of feeders. They can be designed as part of the blanket, thereby avoiding the use of ports.

\footnotetext{
${ }^{1}$ Tore Supra: the values given for the power density is not the maximum power divided by the antenna area, but was calculated from the maximum value obtained on one of the antenna's.
}

Table 1. Power density of a number of ICRF antennas

\begin{tabular}{lllll}
\hline $\begin{array}{l}\text { Experi } \\
\text { ment }\end{array}$ & $\begin{array}{l}\text { number } \\
\text { of } \\
\text { antennas }\end{array}$ & $\begin{array}{l}\text { total } \\
\text { antenna } \\
\text { surface } \\
{\left[\mathrm{m}^{2}\right]}\end{array}$ & $\begin{array}{l}\text { Power } \\
{[\mathrm{MW}]}\end{array}$ & $\begin{array}{l}\text { Power } \\
\text { density } \\
{\left[\mathrm{MW} / \mathrm{m}^{2}\right]}\end{array}$ \\
\hline AUG & 4 & 3.2 & 7.2 & 2.25 \\
TS1 & 3 & 0.9 & 13.2 & 15 \\
JET A0 & 4 & 14.4 & 22 & $\sim 1.5$ \\
JET & 1 & 0.91 & $(2.8)$ & 6.2 \\
ILA2 & & & & \\
\hline ITER & 2 & $\sim 6$ & 20 & $\sim 3.3$ \\
\hline
\end{tabular}

\section{TWA concept}

\subsection{Principle}

A Traveling wave antenna $[1,2]$ is an array of mutually coupled resonant straps connected in parallel, and where the power is fed in at the first and out at the last strap of the array. This becomes an array of resonators where the

2 The maximum power density on the ILA was obtained with only the lower half of the antenna operating. The value for the power density is given for $2.8 \mathrm{MW}$ (on the lower half) extrapolated to the whole antenna. 
current on each of the intermediate straps is induced by mutual coupling (mostly inductive for the ion cyclotron frequency range). The current is decaying from one strap to the other, along the strap array due to power being coupled to the plasma. A circuit of such an array is given in Fig.1 where a resistor is shown in series to each strap to simulate the radiation resistance. The value of this resistance can be computed from the impedance matrix describing the array using ANTITER-II with a realistic plasma density profile in front of the antenna $[3,4]$.

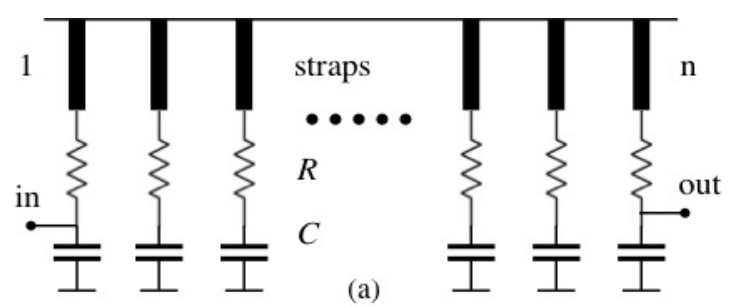

Fig. 1. Finite array of straps. Simplified model.

\subsection{Feeding schemes}

Different feedings schemes are possible for the TWA. Note that the figures are shown as simple examples with four inputs (4-fold symmetry). In practice more inputs would be foreseen.

\subsubsection{Full ring with distributed feeding}

In this case, input feeding lines are connected to a continuous array. The concept is shown in figure 2 , and resulting spectra in figure 3 .

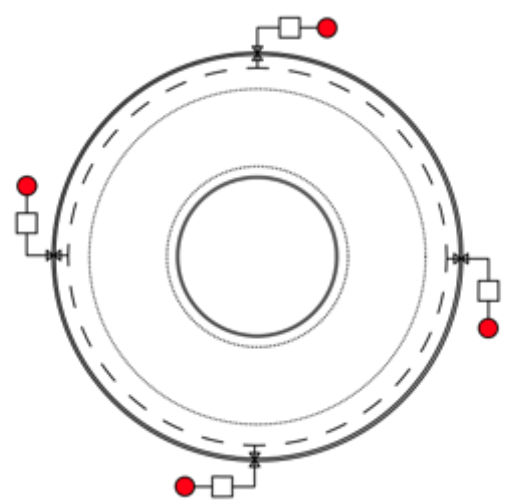

Fig. 2. Example of a ring with 28 straps and distributed feeding at 4 locations.

The concept has the advantage of the largest symmetry and of the smallest number of feeders. It however only works if the symmetry is fully maintained, with all straps and all generators working. This could lead to a reduction of the reliability of the overall system.

The system is not load resilient. A variation of the plasma loading on the antenna, or a failure of one generator, will lead to a change in the input impedance of the neighboring generators causing a significant power imbalance. The result is that both neighboring generators are mismatched and receive a large amount of reflected power leading to the actuation of the safety protection system and a tripping the RF generators. For those reasons, this feeding scheme was not further pursued.

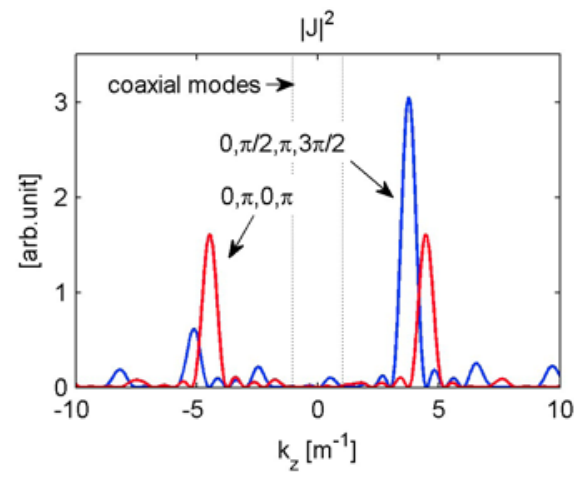

Fig. 3. Spectra of the excitation array current versus the toroidal wave number $k_{z}$ for two phase differences, i.e. $\pi$ and $\pi / 2$, between generators.

\subsubsection{Ring with external resonant feeders}

An alternative option is to use a number of independent TWA sections, fed by resonant rings as shown in Fig. 4 (with four TWA sections). Each resonant ring is connected to a generator and composed by a TWA, a variable coupler, a line stretcher (phase shifter) and a dummy load (see also 2.3 resonant ring feeding scheme). The resulting spectrum is shown in Fig. 5. The sections do not have to be contiguous and can be located in any poloidal position, e.g. on the top or bottom of the ports to avoid obstructing other systems. This allows a greater flexibility than a contiguous ring.

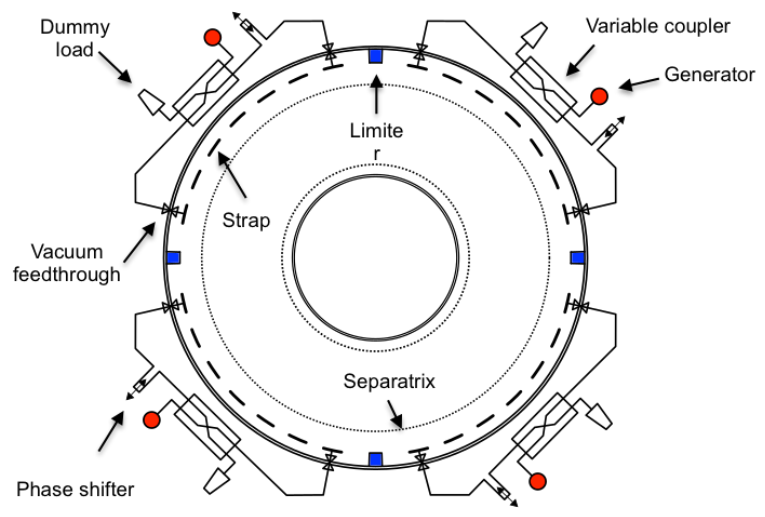

Fig. 4. Example of 4 TWA sections fed by 4 independent resonant rings.

The failure of one TWA section will not lead to a failure of the whole IC system but rather to a small reduction of the radiated power. The example in Fig. 4 is only schematic. In fact, more sections would be foreseen. For 16 sections, following the symmetry of the toroidal field coils, the reduction with the loss of one section would be only $6.25 \%$. Moreover, each TWA section could be designed with a safety margin with respect to its power and voltage capability. An increase in the voltage by $3.3 \%$ on the remaining 15 sections, would compensate for the loss of one of the 16 sections. A majority of the components of the antenna system are located in areas where maintenance does not pose a problem (i.e. outside the cryostat, see Fig.6). 


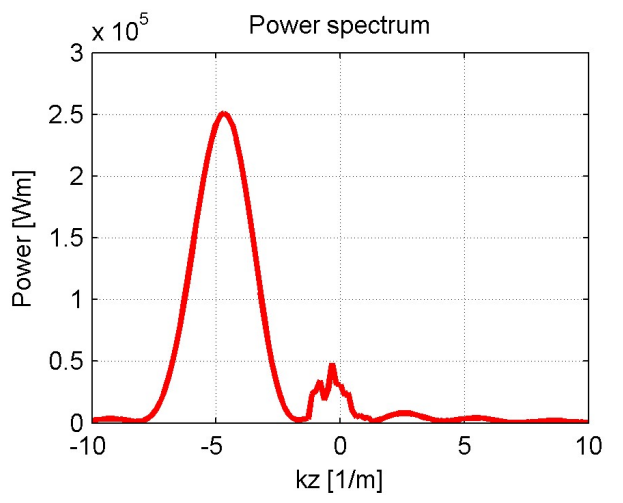

Fig. 5. Indicative spectrum for one of the sections in figure 4.

\subsection{Resonant ring feeding scheme}

The main advantage of using. separate TWA sections (as in 2.2.2) with a resonant ring feeding is that it allows to recirculate the RF power leaving the TWA array [3]. In this way, if one neglects ohmic losses in the antenna system, all the generator power can be deposited to the plasma. The antenna system $[2,3]$ then consists of: one TWA array, two line-stretchers (LSC and LSR) and two hybrids (See Fig. 6). LSC controls the coupling of the variable coupler formed with the two hybrids while LSC controls the resonance condition of the ring. The TWA is the only component inside the vacuum vessel. This increases the RAMI score considerably (as compared to other antenna types with matching components inside the vessel). All the transmission lines are matched so they can be safely operated without the overvoltage and overcurrent, that are typical in unmatched lines. Ohmic losses are thus minimized.

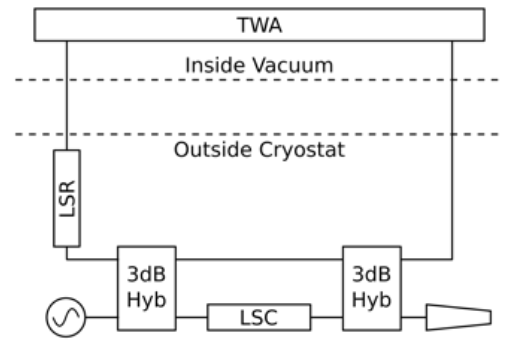

Fig. 6. Traveling wave array antenna fed by a resonant ring. LSC and LSR are line-stretchers (LS) in respectively the coupler part and the resonant part of the ring.

\subsection{Application to DEMO}

A system made of 16 contiguous TWA sectors is proposed, as schematically shown in the artist's view of Fig.7. Each sector is made of two superposed TWA arrays, each containing 8 straps. A detailed view of one TWA array is given in Fig.8.

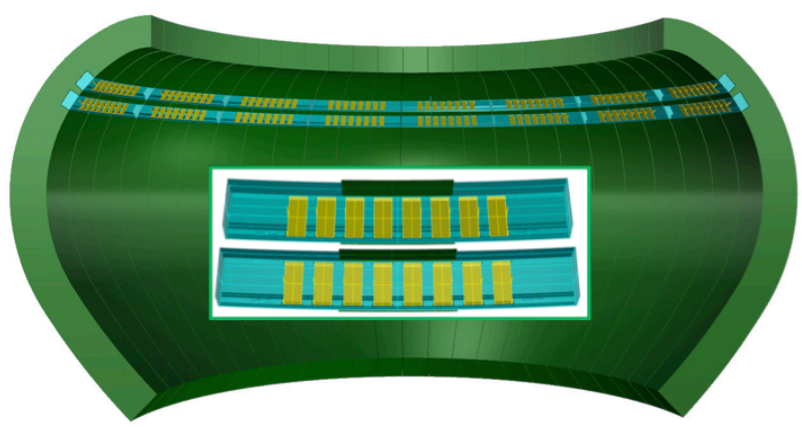

Fig. 7. Artist's view of the 16 sectors with a double $8 \mathrm{~T}$ strap array embedded in the blanket and proposed as a possible ICRF system for DEMO. Half of the machine is shown while in the inset a detailed view of a sector with the two TWA is presented. Whereas the drawings are shown without Faraday screen, it is still open whether or not a FS will be needed.

A summary of the performance of the system is given in Table 2 where the coupled power, the maximum voltage on the straps and the power density per section are listed for different edge density plasma profiles. In the absence of a DEMO reference density profile, computations were performed with the ITER-2010-low density profile [9] (see Fig.9), which represents a "low coupling" profile. For this reason, the profile is moved forward and backward w.r.t. its reference position in order to show the different performances that depend heavily on the antenna-cutoff distance, and thus on the details of the profile.

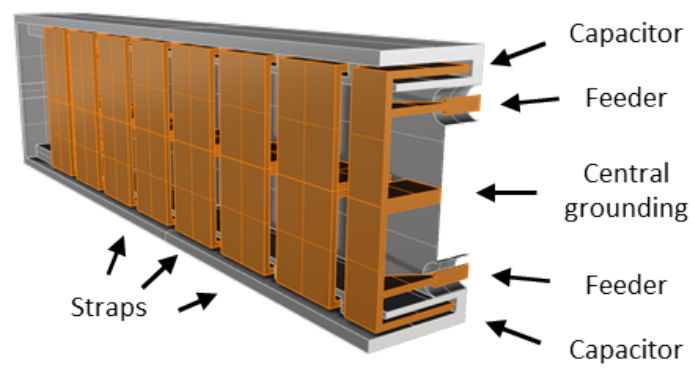

Fig. 8. One T-type TWA array section consisting of 8 T-type straps. Only the first and last strap are connected to feeders (top and bottom).

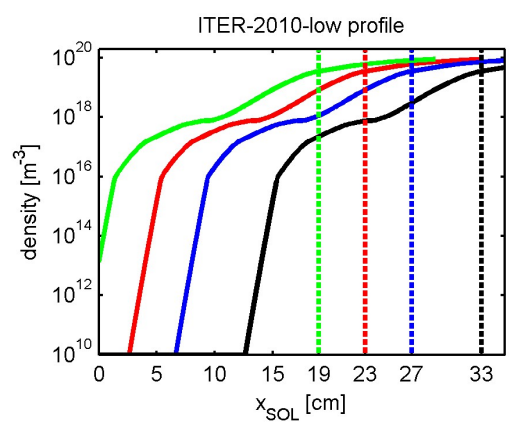

Fig. 9. Different plasma profiles used in the computation. The reference one (red) is the ITER 2010-low profile [9] characterized by a distance between the antenna aperture and last closed magnetic surface of $23 \mathrm{~cm}$. The other profiles are shifted by $-4 \mathrm{~cm}$ (green), $+4 \mathrm{~cm}$ (blue) and $+10 \mathrm{~cm}$ (black). 
The requested power for DEMO (50 MW) could be obtained with a maximum voltage of $15 \mathrm{kV}$ on the antenna structures at a power density of less than $1 \mathrm{MW} / \mathrm{m}^{2}$. As expected, the profile plays a fundamental role in the calculation of the deposited power to the plasma. When displaced by $-4 \mathrm{~cm}$ the power coupled exceeds $70 \mathrm{MW}$ and decreases to $40 \mathrm{MW}$ when displaced by $+4 \mathrm{~cm}$.

\section{Integration in the blanket}

\subsection{Blanket}

Integrating the TWA antenna system in the most recent design for the Helium Cooled Pebble Bed (HCPB) blanket (consisting of radial breeding pins [5]) shows that using the He from the blanket as coolant, the maximum temperatures in the TWA antenna structure are well below $550^{\circ} \mathrm{C}$, which is the maximum allowed for EUROFER. The helium outlet temperature and pressure drop in the antenna cooling circuit are consistent with the Balance of Plant $(\mathrm{BoP})$ requirements of the blanket. Furthermore, cooling of the feeders inside the blanket and upper port area using $\mathrm{He}$ from the blanket combined with radiative cooling of the feeders substantially simplifies the cooling concept and is compatible with remote handling of the blanket segments (See section 3.3). Figures 10a-d show the integration scenario proposed of an antenna in the blanket.

Table 2. Performance of the 16 double T-strap array for different displacements in the edge density profile. The reference profile corresponds to distance from an antenna to the last closed magnetic surface of $23 \mathrm{~cm}$ (see figure 9).

\begin{tabular}{llll}
\hline Profile & $\begin{array}{l}\text { Power } \\
(\mathrm{MW})\end{array}$ & $\begin{array}{l}\text { Voltage } \\
(\mathrm{kV})\end{array}$ & $\begin{array}{l}\text { Power } \\
\text { density } \\
\left(\mathrm{MW} / \mathrm{m}^{2}\right)\end{array}$ \\
\hline$-4 \mathrm{~cm}$ & 72.2 & 15 & 1.16 \\
Ref & 59.5 & 15 & 0.930 \\
$+4 \mathrm{~cm}$ & 40.6 & 15 & 0.635 \\
$+10 \mathrm{~cm}$ & 27.4 & 15 & 0.428 \\
\hline
\end{tabular}

\subsection{Calculation of the Tritium Breeding Ratio (TBR)}

The analysis was based on the most up to date Helium Cooled Pebble Bed (HCPB) blanket design [5] as the reference blanket. To assess the TBR reduction we used a simplified blanket model with the completely homoge- nized interior blanket structure was adopted. The reference TBR without antenna and feeders is 1.2. With antenna and feeders, the TBR decreases to 1.18. The reduction due to the presence of antenna and feeders, assuming $360^{\circ}$ coverage, is thus $\sim 2 \%$ [6]. This small effect can be qualitatively understood. The antenna inserted at the front of the blanket leads to a reduction of the breeder volume, but the related TB loss is partly compensated by an increased tritium production in the less shielded breeder zone behind the antenna. One can also see it in another way (though this is a bit oversimplified): the antenna being mostly empty space, it is as if the breeding region is shifted by the depth of the antenna, and the depth lost for the tritium breeding is the equivalent depth of the antenna at the back of the blanket (where the tritium breeding, which decays exponentially through the blanket, is already low). The effect of the feeders is small because of their small numbers. The results are consistent with previous calculations where only the effect of the antenna was assessed [7].

\subsection{Impact on remote handling}

Electrical RF continuity is needed in the back plane of the antenna for the continuity of the RF currents. A continuous back plane leads to concerns in terms of grounding and forces due to induced currents. In addition, it raises questions on how to satisfy the requirements (i) for separate blanket segments (which are handled individually through the top ports of the machine) and (ii) a continuous back plane covering several segments. The continuity of the RF currents between separate breeding blanket segments could be realized using capacitive elements, but this solution still needs to be investigated.

Because of remote handling, routing the transmission lines through the upper ports would be preferable and is possible both for a TWA antenna located in the upper part of the first wall of DEMO (see figures 10a-d) or near the equatorial plane (which maybe preferred if the emphasis is on heating) On the outside the TL could be connected using flanges, such that there is no need for rewelding and cutting. 

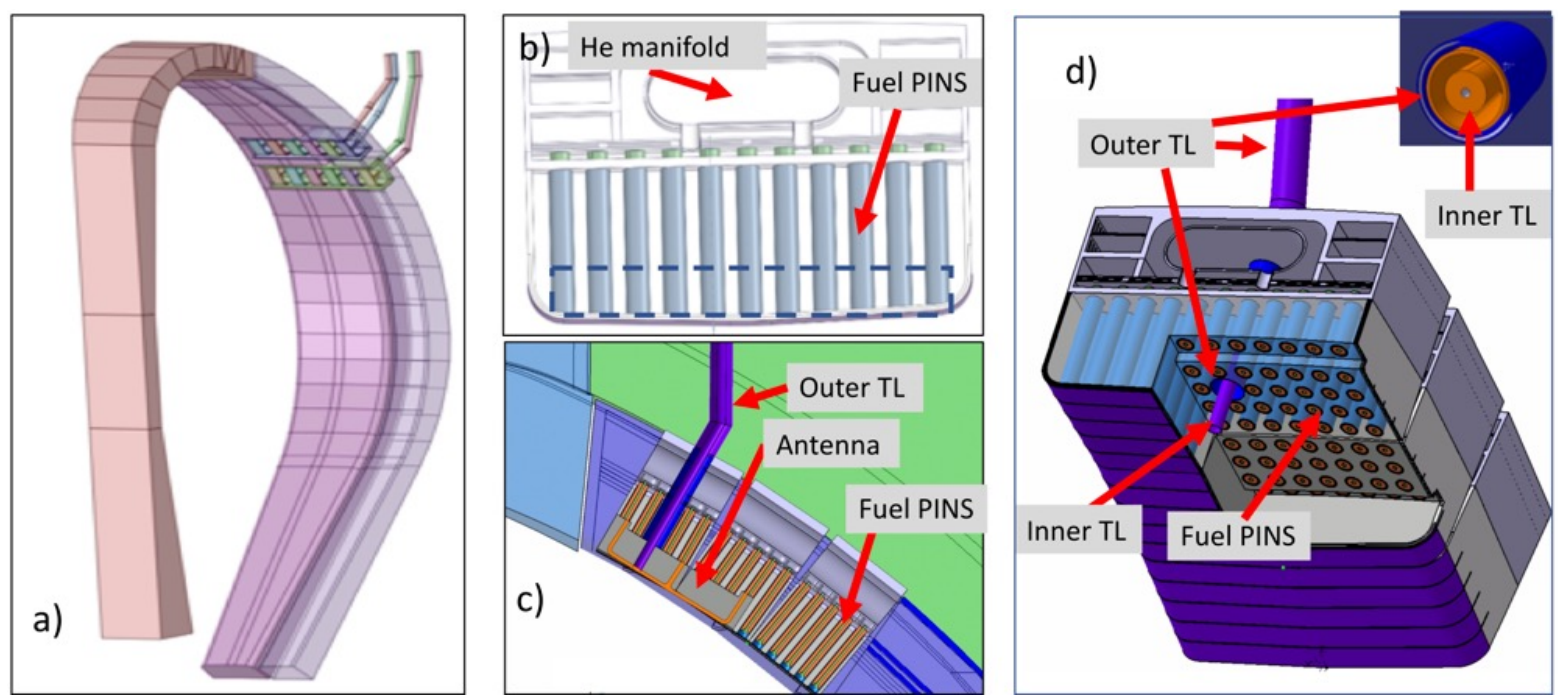

Fig. 10. a) one sector of DEMO $\left(1 / 32=11.25^{\circ}\right)$ with double antenna arrays (radial depth $339 \mathrm{~mm}$ and $1100 \mathrm{~mm}$ poloidal extend) and 4 transmission lines of $100 \mathrm{~mm}$ diameter each, as used for the MCNP calculations; b) radial cut through a breeding blanket, showing the fuel pins and the He cooling manifold, the blued dashed box at the front indicates the location of the antenna; c) poloidal cut through a breeding blanket segment, showing the fuel pins, and one T- type antenna array with a single TL feeder; d) 3-D detailed view of a blanket segment with the fuel pins and the He cooling manifold. It also shows how a TL essentially replaces one fuel pin (antenna not shown).

\section{Summary and concluding remarks}

A TWA with an external resonant feeding scheme is a good candidate for an ICRF system on DEMO. A test on a medium size tokamak is however needed to provide a proof of the concept and is being envisaged [8].

Further alternative concepts, such as an ITER-like, inport antennas are being considered in parallel This concept could have the advantage of a reduced need for integration but would need to operate at higher power densities and voltages.

\section{Acknowledgments}

This work has been carried out within the framework of the EUROfusion Consortium and has received funding from the Euratom research and training programme 2014-2018 under grant agreement No 633053 in the framework of the PPPT programme. The views and opinions expressed herein do not necessarily reflect those of the European Commission.

\section{References}

[1] R. Ragona and A. Messiaen 2016 Nucl. Fusion 56 076009. https://doi.org/10.1088/0029-5515/56/7/076009

[2] C. P. Moeller et al., in Proc. Europhysics Top. Conf. on RF Heating and CD, Brussels BE, 1992, vol 16E, 53.

[3] R.Ragona, $\mathrm{PhD}$ Thesis, UGent, 2018

[4] A. Messiaen and R. Ragona 2017 EPJ Web of conferences 157, 03033 https://doi.org/10.1051/epjconf/201715703033

[5] F. A. Hernandez-Gonzalez et al., An enhanced, near-term HCPB configuration as driver blanket for the EU DEMO, this conference.

[6] A. Bader, EUROFusion Report, 2018

[7] Garcia Perez et al., Fus. Eng. Des.112, (2016), 298-302.

[8] R. Ragona et al., TWA Proof of Principle on WEST, this conference.

[9] Carpentier S. and Pitts R. Areport ITER D33y59M v2,3 (April 2010). 